\title{
Effect of Organic and Conventional Farming on the Activity of Spider Assemblage (Araneae) in Some Medicinal Plants in Fayoum-Egypt
}

\author{
Marguerite A. Rizk; Mona M. Ghallab and A.Y. Zaki \\ Plant Protection Research Institute, A. R. C. Dokki, Giza, Egypt.
}

\begin{abstract}
Spider activity occurred on three medicinal plants (Wormwood, Chrysanthemum and Spearmint) with organic and inorganic fertility was assessed using pitfall traps in Fayoum, Egypt. Community composition of the organic and the conventional cultivation of collected spiders were determined throughout the period of study using the Shannon-Wiener and Simpson Indices of diversity. Totals of 387 and 350 individuals including 20 and 24 species in the organic and the conventional cultivation, respectively, were of higher diversity index in the conventional system. According to Simpson, it was found that organic cultivation included the highest number of dominant species. Most species caught belonged to the family Lycosidae with especial high captures of Wadicosa fidelis and Pardosa species. Sorensen Quotient of Similarity concluded that the two communities were nearly approximate, as they recorded $64 \%$ of similarity. The monthly fluctuation of the total number of spiders showed high population in March.
\end{abstract}

Key words: Chrysanthemum, Conventional cultivation, Organic cultivation, Shannon-Wiener Index, Simpson index, Spearmint, Spider diversity, Wormwood.

\section{INTRODUCTION}

Organic management promotes the development of soil fauna such as earthworms and above ground arthropods, improving the growth conditions of the crop. More abundant predators help to control harmful organisms. Spiders constitute one of the major groups of generalist predators due to their high abundance and predominantly insectivorous feeding habits (Tahir \& Butt, 2009). They play an important predatory role in agro-ecosystem (Nyffeler and Benz, 1987; Nyffeler, 2000a \& b; Symondson et al., 2002). However, crop type had more effect on the activity of spiders than management system. However, several factors affect population density and diversity of spiders. Of these, organic field support a higher abundance of spiders than conventional fields (Feber et al., 1998; Schmidt et al., 2005 and Fuller et al., 2005). (Eyre et al., 2007) found that fertility rather than crop protection management had considerably more influence on the activity of beneficial invertebrates.

The biological diversity in the organic system is important because it contributes in keeping the biological equilibrium, which is essential in an agro ecosystem. It brings about greater stability for the system and consequently fewer problems with diseases and pests (Bettiol et al., 2002). Diversity indices provide more information about community composition than simply species richness that tacking relative abundance in consideration. It is an important tool for biologists trying to understand community structure. It is argued that organic systems are more diverse and therefore more stable resulting in lower incidences of pest and disease problems and increased biodiversity (Lampkin, 1990).
Present study aimed to evaluate the influence of the organic and the conventional cropping systems for three medicinal plants on the activity density and diversity of spiders.

\section{MATERIALS AND METHODS}

\section{Field trial}

A field experiment of $2100 \mathrm{~m}^{2}$ in two different regions in Fayoum governorate for conventional and organic management was conducted. Each region was divided into three equal plots $\left(700 \mathrm{~m}^{2}\right.$ each) cultivated with three medicinal plants; wormwood, Artemisia sp.; Daisy, Chrysanthemum vulgare (L.) and Egyptian spearmint, Mentha niliaca Jacq. The conventional plots were treated with inorganic fertilizers and sprayed with pesticides when appropriate; while the organic plots were fertilized with compost and no sprays were used. Spiders were sampled by using pitfall traps method as described by Southwood (1978) and Slingsby \&Cook (1986). Ten pitfall traps were placed for each plant every ten days. Samples were sorted in the laboratory; collected spiders were kept in glass vials containing $75 \%$ ethyl alcohol with some droplets of glycerin, counted and identified to species level as much as possible.

\section{a- Frequency and abundance values}

The frequency values of the most abundant species were classified into three categories according to the system adopted by Weis Fogh (1948); "Constant species" more than $50 \%$ of the samples, "Accessory species" $25-50 \%$ of the samples and "Accidental species" less than 25\%. On the other hand, the classification of dominance values were done according to Weigmann (1973) system in which the species were divided into five groups based on the 
values of dominance in the sample; Eudominant species (> 30\% individuals), dominant species (10$30 \%$ individuals), subdominant (5-10\% individuals) recedent species (1-5\% individuals) and subrecedent species ( $1 \%$ individuals).

\section{b- Species diversity}

The biodiversity of ground fauna collected were estimated by using equilibrium. Diversity of collected arthropods was determined for samples pooled over one summer season by two different patterns of fertilization. It was measured by diversity index that reflected the number of species (richness) in the samples. Two common indices were computed, Shannon-Wiener index "H" and Simpson index "S". and calculated as described by Ludwig and Reynolds (1988).

$$
\mathrm{H}^{\prime}=-\sum(\mathrm{n} i / \mathrm{n}) \ln (\mathrm{n} i / \mathrm{n}) \text { and } \mathrm{S}=\sum(\mathrm{n} i / \mathrm{n})^{2} .
$$

$n i$ is the number of individuals belonging to the $\mathrm{i}^{\text {th }}$ of "S" taxa in the sample where " $\mathrm{n}$ " is the total number of individuals. "H" is more sensitive to changes in number of species and diversity, while "S" is a dominance index that gives more weight to common or dominant species (Ludwig \& Reynolds, 1988); it highly suggests that the two individuals drawn at random from the population belong to the same species. If the result is high then the probability of both individuals belonging to the same species is high, and as a result the diversity of the community samples might be low.

\section{c- Sorensen quotient of similarity}

To allow a comparison of the two samplings between microhabitats of the two cultivation systems, Sorensen's quotient of similarity (Sorensen, 1948) was used to determine the similarities of spider species composition among the communities, it is:

$$
\mathrm{QS}=2 \mathrm{C} / \mathrm{A}+\mathrm{B} \text {. }
$$

Where $A$ and $B$ are the number of species in samples $\mathrm{A}$ and $\mathrm{B}$, respectively, and $\mathrm{C}$ is the number of species shared by the two samples; QS is the quotient of similarity and ranges from 0 to 1 .

\section{RESULTS AND DISCUSSION}

\section{Spider assemblage}

Collected spiders recorded 737 individuals representing 10 families, 26 genera and 27 species. The 10 families found in the present experiment represent $25 \%$ of the 40 families recorded in Egypt by (El-Hennawy, 2006).

\section{Spiders inhabiting land of organic management}

A total of 387 spider species were caught in the organic cultivation. They were classified in 7 families and 20 genera and 20 species. Juveniles comprised
$26.6 \%$; while adults averaged $73.4 \%$. The sex ratio was 2.9 males: 1 female Table (1). Of the most abundant species, 5 ranked in the top, Wadicosa fidelis (195 individuals), Pardosa sp. $\quad$ (89 individuals), Hogna sp. (35 individuals), Thanatus albini (14 individuals), and Zelotes sp. (12 individuals).

Vegetation type influenced spider abundance as plots of spearmint and daisy plants Table (3) received a high number of spiders, 151 individuals for both plants, while wormwood showed the lowest number, 85 individuals, its natural chemical structure contained within leaves become detergent to common pests and in turn the abundance of spiders was reduced. However, wormwood plant seemed to have a higher diversity than spearmint and daisy plants because it had the greatest number of species recorded $14,12 \& 13$ species, respectively. This result is in accordance with Rizk et al. (2012) who indicated that spider assemblages were highly influenced by variations in plant community structure. Liu et al., 2003 indicated that the density of spiders in the field increase with the increase in plant size and complexity, thus smaller plants has fewer spiders than tall ones.

\section{Spiders inhabiting land of conventional management}

A total of 350 spider individuals were cached in the conventional cultivation. They were represented by 10 families and 23 genera and 23 species. Juveniles comprised $28.3 \%$; while adults averaged $71.7 \%$. The sex ratio was 2.4 males: 1 female (Table, 2). The most dominant species was Wadicosa fidelis (218 individuals), Pardosa sp. (46 individuals), Steatoda erigoniformis (13 individuals) and Hogna sp. (11individuals).

Daisy plants supported a higher abundance of spiders, 137 individuals and two egg sacs; while spearmint and wormwood plants had 118 and 95 individuals, respectively. However wormwood plant had the fewest number of spiders in the conventional management but rather of high diversity which recorded 16 species; while daisy and spearmint recorded 15 and 11 individuals, respectively. Schmidt et al., 2005, found that most activity density of spider was in conventional management crops.

Members of Theridiidae and Linyphidae were more active in conventional management while Lycosidae and Gnaphosidae preferring organic management; this result was in accordance with that of Eyre et al. (2008).

\section{Species richness}

Among the 23 species of spiders collected during the study, 20 species of 7 families were recorded in 
Table (1): Species richness of spiders inhabiting land of organic management

\begin{tabular}{|c|c|c|c|c|c|c|c|c|c|c|c|c|c|c|c|}
\hline \multirow{2}{*}{ Scientific name } & \multicolumn{3}{|c|}{ Wormwood } & \multicolumn{3}{|c|}{ Daisy } & \multicolumn{3}{|c|}{ Spearmint } & \multicolumn{3}{|c|}{$\Sigma$} & \multirow{2}{*}{$\Sigma$} & \multirow{2}{*}{ Total } & \multirow{2}{*}{$\%$} \\
\hline & $\hat{0}$ & $q$ & $\mathrm{j}$ & $\hat{3}$ & 9 & $\mathrm{j}$ & $\hat{0}$ & 9 & $\mathrm{j}$ & $\hat{0}$ & $q$ & $\mathrm{j}$ & & & \\
\hline Lycosidae & 2 & & 1 & & & & 1 & & & 3 & & 1 & 4 & \multirow{4}{*}{$323+3 \boldsymbol{\Delta}$} & \multirow{4}{*}{83.5} \\
\hline Wadicosa fidelis & 25 & 8 & 1 & 32 & 17 & 40 & 41 & $20+\boldsymbol{\Delta}$ & 11 & 98 & $45+\boldsymbol{\Lambda}$ & 52 & $195+\boldsymbol{\Delta}$ & & \\
\hline Pardosa sp. & 11 & $7+\boldsymbol{\Delta}$ & 2 & 8 & 3 & 6 & 37 & 7 & 8 & 56 & $17+\boldsymbol{\Delta}$ & 16 & $89+\boldsymbol{\Lambda}$ & & \\
\hline Hogna ferox & 4 & 1 & & 2 & $5+\boldsymbol{\Delta}$ & 19 & 3 & 1 & & 9 & $7+\boldsymbol{\Delta}$ & 19 & $35+\boldsymbol{\Delta}$ & & \\
\hline Salticidae & & & & & & & & & 1 & & & 1 & 1 & \multirow{3}{*}{6} & \multirow{3}{*}{1.5} \\
\hline Phlegra flavipes & 4 & & & & & & & & & 4 & & & 4 & & \\
\hline Heliophanillus fulgens & 1 & & & & & & & & & 1 & & & 1 & & \\
\hline Philodromidae & & & & & & & & & & & & & & \multirow{2}{*}{14} & \multirow{2}{*}{3.6} \\
\hline Thanatus albini & 2 & & 1 & 5 & & & 4 & 1 & 1 & 11 & 1 & 2 & 14 & & \\
\hline Eutichuridae & & & & & & & & & & & & & & \multirow{2}{*}{3} & \multirow{2}{*}{0.8} \\
\hline Cheiracanthium sp. & & & 2 & 1 & & & & & & 1 & & 2 & 3 & & \\
\hline Theridiidae & & & & & & & & & & & & & & \multirow{4}{*}{8} & \multirow{4}{*}{2.1} \\
\hline Steatoda erigoniformis & 1 & & & 2 & 1 & & 2 & & 1 & 5 & 1 & & 6 & & \\
\hline Enoplognatha gemina & & & & 1 & & & & & & 1 & & & 1 & & \\
\hline Kochiura aulica & & & & 1 & & & & & & 1 & & & 1 & & \\
\hline Gnaphosidae & & & 2 & & & & & & 1 & & & 3 & 3 & \multirow{4}{*}{20} & \multirow{4}{*}{5.2} \\
\hline Zelotes laetus & 3 & & 3 & & & 1 & 5 & & & 8 & & 4 & 12 & & \\
\hline Micaria dives & 1 & 1 & & 1 & 1 & & & & & 2 & 2 & & 4 & & \\
\hline Trachyzelotes jaxartensis & & & & 1 & & & & & & 1 & & & 1 & & \\
\hline Linyphidae & & & & 2 & 1 & & & & 1 & 2 & 1 & 1 & 4 & \multirow{4}{*}{13} & \multirow{4}{*}{3.4} \\
\hline Mermessus denticulatus & & & & & & & 2 & 1 & 1 & 2 & 1 & 1 & 4 & & \\
\hline Sengletus extricatus & 1 & & & & & & & & & 1 & & & 1 & & \\
\hline G1 & 1 & & & 1 & & & 1 & & 1 & 3 & & 1 & 4 & & \\
\hline$\Sigma$ & 56 & $17+\Delta$ & 12 & 58 & $27+\boldsymbol{\Delta}$ & 66 & 97 & $29+\boldsymbol{\Delta}$ & 25 & 211 & $73+3 \mathbf{\Delta}$ & 103 & $387+3 \boldsymbol{\Delta}$ & $387+3 \boldsymbol{\Delta}$ & \\
\hline Total & & $+\boldsymbol{\Delta}$ & & & $151+\boldsymbol{\Delta}$ & & & $151+\boldsymbol{\Delta}$ & & & $387+3 \mathbf{\Delta}$ & & & & \\
\hline
\end{tabular}

$\boldsymbol{\Lambda}$ : Egg sac

Table (2): Species richness of spiders inhabiting land of conventional management

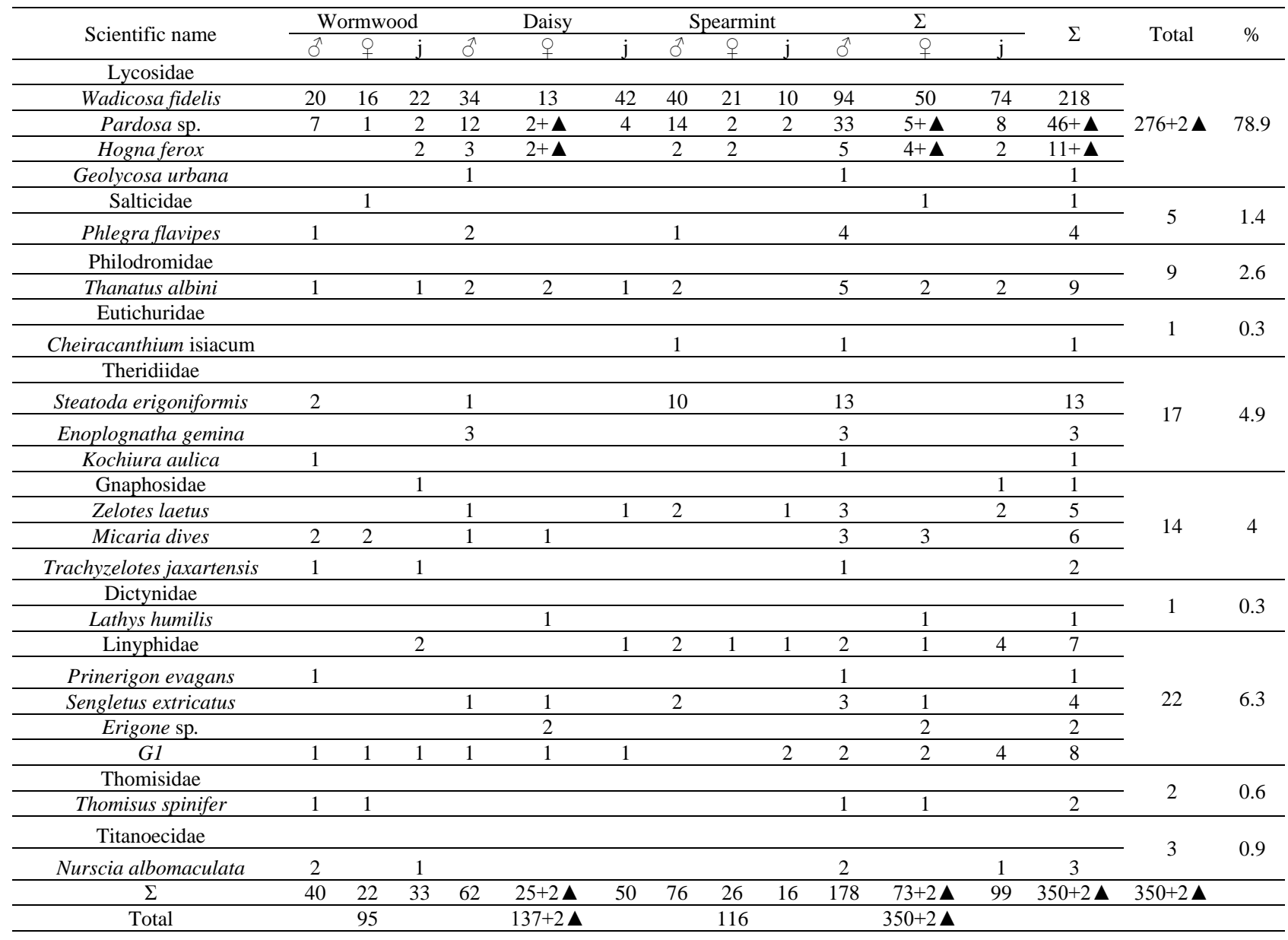

A : Egg sac 
Table (3): Occurrence of spiders in organic and conventional managements

\begin{tabular}{|c|c|c|c|c|c|c|c|c|}
\hline \multirow[b]{2}{*}{ Scietific name } & \multicolumn{2}{|c|}{ Wormwood } & \multicolumn{2}{|c|}{ Daisy } & \multicolumn{2}{|c|}{ Spearmint } & \multicolumn{2}{|c|}{ Total } \\
\hline & $\mathrm{A}$ & $\mathrm{B}$ & $\mathrm{A}$ & $\mathrm{B}$ & $\mathrm{A}$ & $\mathrm{B}$ & $\mathrm{A}$ & $\mathrm{B}$ \\
\hline Lycosidae & 3 & & & & 1 & & 4 & \\
\hline Wadicosa fidelis & 34 & 58 & 89 & 89 & $72+\boldsymbol{\Delta}$ & 71 & $195+\boldsymbol{\Lambda}$ & 218 \\
\hline Pardosa sp. & $20+\boldsymbol{\Delta}$ & 10 & 17 & $18+\mathbf{\Delta}$ & 52 & 18 & $89+\boldsymbol{\Lambda}$ & $46+\boldsymbol{\Delta}$ \\
\hline Hogna ferox & 5 & 2 & $26+\Delta$ & $5+\boldsymbol{\Delta}$ & 4 & 4 & $35+\mathbf{\Delta}$ & $11+\mathbf{\Delta}$ \\
\hline Geolycosa urbana & & & & 1 & & & & 1 \\
\hline Salticidae & & 1 & & & 1 & & 1 & 1 \\
\hline Phlegra flavipes & 4 & 1 & & 2 & & 1 & 4 & 4 \\
\hline Heliophanillus fulgens & 1 & & & & & & 1 & \\
\hline \multicolumn{9}{|l|}{ Philodromidae } \\
\hline Thanatus albini & 3 & 2 & 5 & 5 & 6 & 2 & 14 & 9 \\
\hline \multicolumn{9}{|l|}{ Eutichuridae } \\
\hline Cheiracanthium isiacum & & & & & & 1 & & 1 \\
\hline Cheiracanthium sp. & 2 & & 1 & & & & 3 & \\
\hline \multicolumn{9}{|l|}{ Theridiidae } \\
\hline Steatoda erigoniformis & 1 & 2 & 3 & 1 & 2 & 10 & 6 & 13 \\
\hline Enoplognatha gemina & & & 1 & 3 & & & 1 & 3 \\
\hline Kochiura aulica & & 1 & 1 & & & & 1 & 1 \\
\hline Gnaphosidae & 2 & 1 & & & 1 & & 3 & 1 \\
\hline Zelotes laetus & 6 & & 1 & 2 & 5 & 3 & 12 & 5 \\
\hline Micaria dives & 2 & 4 & 2 & 2 & & & 4 & 6 \\
\hline Trachyzelotes jaxartensis & & 2 & 1 & & & & 1 & 2 \\
\hline \multicolumn{9}{|l|}{ Dictynidae } \\
\hline Lathys humilis & & & & 1 & & & & 1 \\
\hline Linyphidae & & 2 & 3 & 1 & 1 & 4 & 4 & 7 \\
\hline Prinerigon evagans & & 1 & & & & & & 1 \\
\hline Sengletus extricatus & 1 & & & 2 & & 2 & 1 & 4 \\
\hline Erigone sp. & & & & 2 & & & & 2 \\
\hline Mermessus denticulatus & & & & & 4 & & 4 & \\
\hline G1 & 1 & 3 & 1 & 3 & 2 & 2 & 4 & 8 \\
\hline \multicolumn{9}{|l|}{ Thomisidae } \\
\hline Thomisus spinifer & & 2 & & & & & & 2 \\
\hline \multicolumn{9}{|l|}{ Titanoecidae } \\
\hline Nurscia albomaculata & & 3 & & & & & 0 & 3 \\
\hline Total & $85+\Delta$ & 95 & $151+\Delta$ & $137+2 \Delta$ & $151+\Delta$ & 118 & $387+3 \Delta$ & $350+2 \Delta$ \\
\hline
\end{tabular}

Table (4): The dominance-frequency relationship of spider communities in organic land

\begin{tabular}{|c|c|c|c|c|c|c|c|c|c|c|c|c|c|c|c|}
\hline \multirow{2}{*}{ Scientific name } & \multicolumn{5}{|c|}{ Wormwood } & \multicolumn{5}{|c|}{ Chrysanthemum } & \multicolumn{5}{|c|}{ Spearmint } \\
\hline & Total & sp.\% & Dom. & F.\% & Freq. & Total & sp.\% & Dom. & F.\% & Freq. & Total & sp.\% & Dom. & F.\% & Freq. \\
\hline Lycosidae & 3 & 3.5 & $\mathrm{R}$ & \multirow{4}{*}{72.9} & \multirow{4}{*}{ C } & & & & \multirow{4}{*}{87.4} & \multirow{4}{*}{ C } & 1 & 0.7 & $\mathrm{sr}$ & \multirow{4}{*}{84.9} & \multirow{4}{*}{ C } \\
\hline Wadicosa fidelis & 34 & 40 & $\mathrm{E}$ & & & 89 & 58.9 & $\mathrm{E}$ & & & 72 & 47.4 & $\mathrm{E}$ & & \\
\hline Pardosa sp. & 20 & 23.5 & $\mathrm{D}$ & & & 17 & 11.3 & $\mathrm{D}$ & & & 52 & 34.2 & $\mathrm{E}$ & & \\
\hline Hogna ferox & 5 & 5.9 & sd & & & 26 & 17.2 & $\mathrm{D}$ & & & 4 & 2.6 & $\mathrm{R}$ & & \\
\hline Salticidae & & & & \multirow{3}{*}{5.9} & \multirow{3}{*}{ A } & & & & & & 1 & 0.7 & $\mathrm{sr}$ & \multirow{3}{*}{0.7} & \multirow{3}{*}{ A } \\
\hline Phlegra flavipes & 4 & 4.7 & $R$ & & & 0 & 0 & & & & 0 & 0 & & & \\
\hline Heliophanillus fulgens & 1 & 1.2 & $R$ & & & 0 & 0 & & & & 0 & 0 & & & \\
\hline Philodromidae & & & & \multirow{2}{*}{3.5} & \multirow{2}{*}{ A } & & & & \multirow{2}{*}{3.3} & \multirow{2}{*}{ A } & & & & \multirow{2}{*}{3.9} & \multirow{2}{*}{ A } \\
\hline Thanatus albini & 3 & 3.5 & $\mathrm{R}$ & & & 5 & 3.3 & $\mathrm{R}$ & & & 6 & 3.9 & $\mathrm{R}$ & & \\
\hline Eutichuridae & & & & \multirow{2}{*}{2.4} & \multirow{2}{*}{ A } & & & & \multirow{2}{*}{0.6} & \multirow{2}{*}{ A } & & & & \multirow{2}{*}{0} & \multirow{2}{*}{ A } \\
\hline Cheiracanthium sp. & 2 & 2.4 & $R$ & & & 1 & 0.6 & $\mathrm{sr}$ & & & 0 & 0 & & & \\
\hline Theridiidae & & & & & & & & & & & & & & & \\
\hline Steatoda erigoniformis & 1 & 1.2 & $\mathrm{R}$ & & $A$ & 3 & 1.9 & $\mathrm{R}$ & & & 3 & 1.9 & $\mathrm{R}$ & & \\
\hline Enoplognatha gemina & 0 & & & 1.2 & A & 1 & 0.6 & $s r$ & 3.3 & A & 0 & 0 & & 1.9 & A \\
\hline Kochiura aulica & 0 & & & & & 1 & 0.6 & $s r$ & & & 0 & 0 & & & \\
\hline Gnaphosidae & 2 & 2.4 & $R$ & & & & & & & & 1 & 0.7 & $\mathrm{Sr}$ & & \\
\hline Zelotes laetus & 6 & 7.1 & sd & & & 1 & 0.6 & $\mathrm{sr}$ & & & 5 & 3.3 & $\mathrm{R}$ & & \\
\hline Micaria dives & 2 & 2.4 & $\mathrm{R}$ & 11.8 & A & 2 & 1.3 & $\mathrm{R}$ & 2.7 & A & 0 & 0 & & 3.9 & A \\
\hline Trachyzelotes jaxartensis & 0 & 0 & & & & 1 & 0.6 & $\mathrm{sr}$ & & & 0 & 0 & & & \\
\hline Linyphidae & & & & & & 3 & 1.9 & $\mathrm{R}$ & & & 1 & 0.7 & $\mathrm{sr}$ & & \\
\hline Mermessus denticulatus & 0 & 0 & & & & 0 & 0 & & & & 4 & 2.6 & $\mathrm{R}$ & & \\
\hline Sengletus extricatus & 1 & 1.2 & $\mathrm{R}$ & 2.4 & A & 0 & 0 & & 2.7 & A & 0 & 0 & & 4.6 & A \\
\hline G1 & 1 & 1.2 & $R$ & & & 1 & 0.6 & $\mathrm{sr}$ & & & 2 & 1.3 & $\mathrm{R}$ & & \\
\hline$\Sigma$ & 85 & & & & & 151 & & & & & 152 & & & & \\
\hline Frequency (abundance), by W & Fog & & & & & ninance, & y Weig & ann & & & & & & & \\
\hline$>50 \%=$ Constant $(\mathrm{C})$ & & & & & & $\%=\mathrm{Eu}$ & ominant & (E) & & & & $5 \% \mathrm{Re}$ & edent $(\mathrm{F}$ & & \\
\hline $25-50 \%=$ Accessory ( ac ) & & & & & & $30 \%=\mathrm{I}$ & ominant & (D) & & & & $b=\mathrm{Sub}$ & ecedent ( & & \\
\hline$>25 \%=\operatorname{Accidental}(\mathrm{A})$ & & & & & $5-1$ & $\%=\mathrm{Sut}$ & lominan & $(\mathrm{sd})$ & & & & & & & \\
\hline
\end{tabular}


Table (5): The dominance-frequency relationship of spider communities in conventional land

\begin{tabular}{|c|c|c|c|c|c|c|c|c|c|c|c|c|c|c|c|}
\hline \multirow{2}{*}{ Scientific name } & \multicolumn{5}{|c|}{ Wormwood } & \multicolumn{5}{|c|}{ Chrysanthemum } & \multicolumn{5}{|c|}{ Spearmint } \\
\hline & Total & sp.\% & Dom. & F.\% & Freq. & Total & sp.\% & Dom. & F.\% & Freq. & Total & sp.\% & Dom. & F.\% & Freq. \\
\hline \multicolumn{16}{|l|}{ Lycosidae } \\
\hline Wadicosa fidelis & 58 & 61.1 & $\mathrm{E}$ & \multirow{4}{*}{73.7} & \multirow{4}{*}{$\mathrm{C}$} & 89 & 67.9 & $\mathrm{E}$ & \multirow{4}{*}{82.3} & \multirow{4}{*}{$\mathrm{C}$} & 71 & 61.2 & $\mathrm{E}$ & \multirow{4}{*}{80.1} & \multirow{4}{*}{$\mathrm{C}$} \\
\hline Pardosa sp. & 10 & 16.5 & Dom. & & & 18 & 13.1 & Dom. & & & 18 & 15.5 & Dom. & & \\
\hline Hogna ferox & 2 & 2.1 & $\mathrm{R}$ & & & 5 & 3.6 & $\mathrm{R}$ & & & 4 & 3.4 & $\mathrm{R}$ & & \\
\hline Geolycosa urbana & 0 & 0 & & & & 1 & 0.7 & $\mathrm{sr}$ & & & 0 & 0 & & & \\
\hline Salticidae & 1 & 1.1 & $\mathrm{R}$ & \multirow{2}{*}{2.2} & \multirow{2}{*}{ A } & & & & \multirow{2}{*}{1.5} & \multirow{2}{*}{ A } & 1 & 0.9 & $\mathrm{sr}$ & \multirow{2}{*}{4.3} & \multirow{2}{*}{ A } \\
\hline Phlegra flavipes & 1 & 1.1 & $\mathrm{R}$ & & & 2 & 1.5 & $\mathrm{R}$ & & & 4 & 3.4 & $\mathrm{R}$ & & \\
\hline Philodromidae & & & & \multirow{2}{*}{2.1} & \multirow{2}{*}{ A } & & & $\mathrm{R}$ & \multirow{2}{*}{3.6} & \multirow{2}{*}{ A } & & & & \multirow{2}{*}{7.8} & \multirow{2}{*}{ A } \\
\hline Thanatus albini & 2 & 2.1 & $\mathrm{R}$ & & & 5 & 3.6 & & & & 9 & 7.8 & sd & & \\
\hline Eutichuridae & & & & & & & & & \multirow{2}{*}{\multicolumn{2}{|c|}{0}} & & & & \multirow{2}{*}{0.9} & \\
\hline Cheiracanthium isiacum & 0 & 0 & & & & 0 & 0 & & & & 1 & 0.9 & $\mathrm{sr}$ & & A \\
\hline Theridiidae & & & & & & & & & & & & & & & \\
\hline Steatoda erigoniformis & 2 & 2.1 & $\mathrm{R}$ & & & 1 & 0.7 & $\mathrm{sr}$ & & & 10 & 8.6 & sd & & \\
\hline Enoplognatha gemina & 0 & 0 & & 3.2 & A & 3 & 2.2 & $\mathrm{R}$ & 2.9 & A & 0 & 0 & & 8.6 & A \\
\hline Kochiura aulica & 1 & 1.1 & $\mathrm{R}$ & & & 0 & 0 & & & & 0 & 0 & & & \\
\hline Gnaphosidae & 1 & 1.1 & & & & & & & & & & & & & \\
\hline Zelotes laetus & 0 & 0 & & 74 & $A$ & 2 & 1.5 & $\mathrm{R}$ & 3 & $A$ & 3 & 2.6 & $\mathrm{R}$ & 26 & $A$ \\
\hline Micaria dives & 4 & 4.2 & $\mathrm{R}$ & 1.4 & A & 2 & 1.5 & $\mathrm{R}$ & 3 & A & 0 & 0 & & 2.0 & A \\
\hline Trachyzelotes jaxartensis & $\begin{array}{ll}s \quad 2 \\
\end{array}$ & 2.1 & $\mathrm{R}$ & & & 0 & 0 & & & & 0 & 0 & & & \\
\hline Dictynidae & & & & & & & & & & & & & & & \\
\hline Lathys humilis & 0 & 0 & & & & 1 & 0.7 & $\mathrm{sr}$ & & & 0 & 0 & & & \\
\hline Linyphidae & 2 & 2.1 & $\mathrm{R}$ & & & 1 & 0.7 & $\mathrm{sr}$ & & & 4 & 3.4 & $\mathrm{R}$ & & \\
\hline Prinerigon evagans & 1 & 1.1 & $\mathrm{R}$ & & & 0 & 0 & & & & 0 & 0 & & & \\
\hline Sengletus extricatus & 0 & 0 & & 6.4 & A & 2 & 1.5 & $\mathrm{R}$ & 5.9 & A & 2 & 1.7 & $\mathrm{R}$ & 6.8 & A \\
\hline Erigone sp. & 0 & 0 & & & & 2 & 1.5 & $\mathrm{R}$ & & & 0 & 0 & & & \\
\hline G1 & 3 & 3.2 & $\mathrm{R}$ & & & 3 & 2.2 & $\mathrm{R}$ & & & 2 & 1.7 & $\mathrm{R}$ & & \\
\hline Thomisidae & & & & & & & & & & & & & & & \\
\hline Thomisus spinifer & 2 & 2.1 & $\mathrm{R}$ & 2.1 & A & 0 & 0 & & & & 0 & 0 & & & \\
\hline Titanoecidae & & & & & $A$ & & & & & & & & & & \\
\hline Nurscia albomaculata & 1 & 1.1 & $\mathrm{R}$ & 1.1 & A & 0 & 0 & & & & 0 & 0 & & & \\
\hline$\Sigma$ & 95 & & & & & 137 & & & & & 113 & & & & \\
\hline
\end{tabular}

organic system and 23 species of 10 families in conventional system. A total of 14 species had common occurrence in both plants. Families Dictynidae, Thomisidae and Titanoecidae were absent in land treated with compost as organic system.

Table (1\&2) showed that the family Lycosidae was represented by $323 \& 276$ individuals and $3 \& 2$ in organic and conventional system, respectively. It was the only dominant family, as it comprised 83.5 and $78.9 \%$ of the total catch in organic and conventional cultivation, respectively. Bengtsson et al. (2005) indicated this result which found that organic farming usually increase species richness, having an average 301 higher species richness than conventional farming system; the same result was recorded by Schmidt et al., 2005 who recorded higher spider densities in organic field that being favorable habitat for spiders. Also Tahir \& Butt, 2009 found that Lycosidae was the only dominant family, comprising $77.37 \%$ of the total catch. Moreover, Ostman et al. (2003) indicated this result in which the organic management where agrochemical application prohibited diversity of spider and provided more complex and diverse physical milieu which gave spiders more protection from natural enemies and improved microhabitat as well. Also, Oberg (2007) indicated that organic practice added diversity to the soil structure and increased the abundance of prey and in turn abundance of spiders.

\section{Frequency and abundance values}

Tables (4\&5) showed the frequency and abundance values of the most abundant spiders. Family Lycosidae was considered "constant" according to Weis Fog system, in the two types of cultivations organic and conventional, occupied 83.5 and $78.9 \%$, respectively; also, considered "constant" in the three cultivated plants, Wormwood, Daisy and Spearmint. Members of this family: Wadicosa fidelis and Pardosa sp. ranged between "Eudominant" and "Dominant" according to Weigmann classification of dominance. Our results agreed with Shuang-lin and Bo-ping (2006) who indicated that Lycosidae, was the dominant family and occupied more than $60 \%$ of individuals' community. Moreover, Rizk et al. (2012) indicated that members of Lycosidae were represented by three common species: Wadicosa fidelis, Pardosa injucanda and Pardosa sp. and all their developmental structures were collected by pitfall traps below the four plants Spearmint, Castorbean, Roselle (Karkadi) and Red pepper.

On the other hand, all the remaining families were "Accidental" while their members ranged between Recedent and Subrecedent except of Zelotes laetus of the family Gnaphosidae for organic cultivation and Steatoda erigoniformis in the family Theridiidae in the conventional cultivation being Subdominant. This 
result was in accordance with that of Eyre et al., 2008 who stated that linyphid species preferring plants in conventional cultivation and larger Lycosid, Philodromid and Gnaphosid species favoring the organic cultivation.

\section{Species diversity}

Table (6) compared the biodiversity of collected spiders in different vegetations between organic and conventional cultivation by using Shannon-Wiener "H" and Simpson "S" Indices of diversity.

The vegetations of different plants varied in their spider richness. The collected spiders on plants treated with compost was the highest (recorded 151 individuals, in Daisy and Spearmint) except of Wormwood plants was higher in conventional cultivation than organic.

According to Shannon-Wiener "H" Index, the Wormwood of conventional cultivation recorded the highest value 1.05 of $16 \mathrm{sp}$. and 8 families; while daisy of organic cultivation recorded the smallest value 0.57 of $13 \mathrm{sp}$. and 6 families), so we can say that wormwood plants had a higher diversity index and daisy had a lower diversity index . Similarly, the values calculated for other cultivation described the different species diversity index for each group.

According to Simpson Index, which reflected the measure of dominance, it was found that the Daisy and Spearmint plants included the highest number of dominant species in organic cultivation, recorded 132 \& 129 individuals of Lycosid members, respectively. Our results are in good agreement with Bettiol et al., 2002 who assumed that the number of collembolans found in the organic system was three times as high as that in the conventional system.

\section{Similarity of species}

Species richness of spiders collected from organic system (387 individuals) was greater than that of conventional system (350 individuals); while the number of spider species was greater in conventional (24 species) than that in organic (20 species). Among

Table (6): Estimation of Shannon-Wiener and Simpson Indices of spider diversity in land of organic and traditional managements

\begin{tabular}{lccccccc}
\hline \multirow{2}{*}{ Type of index } & \multicolumn{3}{c}{$\begin{array}{c}\text { Organic } \\
\text { management }\end{array}$} & \multicolumn{3}{c}{$\begin{array}{c}\text { Traditional } \\
\text { management }\end{array}$} \\
\cline { 2 - 8 } & A & B & C & A & B & C \\
\hline Shannon-Wiener Index & 0.99 & 0.57 & 0.62 & 1.05 & 0.75 & 0.85 \\
\hline Simpson Index & 0.55 & 0.82 & 0.73 & 0.55 & 0.69 & 0.63 \\
\hline A: Wormwood (Artemisia sp.), & & & & \\
B: Daisy (Chrysanthemum vulgare), \\
C: Spearmint (Mentha niliaca)
\end{tabular}

the 27 genera obtained, 24 species were catched from conventional land and 20 in organic land, and the common genera were 14 (Table 3). To allow a comparison between the two habitats, Sorensen's Quotient of similarity (QS) for the two communities was 0.67 ; it is concluded that the two communities are slightly different, as they recorded $64 \%$ of similarity.

\section{Monthly fluctuation of spider population}

Monthly counts of spiders collected from the organic and conventional cultivations, between October and first week of May, occurred in high abundance in March recorded 109 individuals and 3 egg sacs for the organic while respective numbers in conventional cultivation was 88 individuals and one egg sac.

Several authors reported that the winter was the lowest abundance for spider while in summer its population density of spider increased; Rizk et al. (2012) indicated that spiders appeared in few numbers in early summer on the four different plants Spearmint, Castorbean, Roselle (Karkadi), and Red pepper and the population density gradually increased showing a peak in June. In general, this data indicated that spiders were active during summer months.

In conclusion, This study exposing that biodiversity of spider is impacted by different management strategies, organic and conventional. So, vegetation architecture and type of fertility are known to affect spider activity. The diversity Indices account for some species are rare and others being common, they serve as variable tools that enable biologists to quantify diversity in a community and describe its numerical structure. It was found in our study that there was considerably more activity in organic plots for most species with the least on the conventional plots. In general, spider abundance, were highest in the organic management plants while conventional cultivation plants had higher species diversity. These results go in line with Eyre et al. (2008) and in contrast to other reports.

\section{ACKNOWLEDGMENTS}

The authors gratefully acknowledge Mr. Hisham K. EL-Hennawy, Arachnology Specialist (spiders and scorpions) for revising the identification of spiders. The study was made possible through a grant from the supported research create, Agriculture Research Center, Ministry of Agriculture.

\section{REFERENCES}

Bettiol, W.; Ghini, R.; Galvao, J. A. H.; Ligo, M. A. V. and Mineiro, J. L. C. 2002. Soil organisms in organic and conventional cropping systems. Sci. 
agric. (Piracicaba, Braz.), 59 (3) Piracicaba July/Sept.

Bengtsson, J.; Ahnström, J. and Weibull, A. C. 2005. The effects of organic agriculture on biodiversity and abundance: a meta-analysis. Journal of Applied Ecology, 42: 261-269.

El-Hennawy, H. K. 2006. A list of Egyptian spiders. Serket, 10 (2): 65- 76.

Eyre, M. D.; Shotton, P. N. and Leifert, C. 2008. Spider (Araneae) species activity, crop type and management factors in an extensive plot trial. Poster at: Cultivating the Future, Based on Science: $2^{\text {nd }}$ Conference of the International Society of Organic Agriculture Research ISOFAR, Modena, Italy. Archieved at http// orgprints.org/11479/1/Eyre_11479_ed.doc.

Feber. R. E.; Bell, J.; Johnson, P.J.; Firbank, L.G. and Macdonald, D.W. 1998. The effects of organic farming on surface-active spider assemblages in wheat in Southern England. UK. J. Arachnol., 26: $190-202$.

Fuller, R. J.; Norton, L. R.; Feber, R. E.; Johnson, P. J.; Chamberlain, D. E.; Joys, A. C.; Mathews, F.; Stuart, R. C.; Townsend, M. C.; Manley, W. J.; Wolfe, M. S.; Macdonald, D.W. and Firbank, L. G. 2005. Benefits of organic farming to biodiversity vary among taxa. Biol. Lett., 1: 431434.

Lampkin, N. 1990. Organic farming. Farming Press Book Ipswich, U. K., 720 pp.

Liu, W. X.; Hou, M. L.; Wan, F. H and Wang, F. L. 2003. Temporal and spatial niche dynamics of spiders and their control effects on cotton bollworms in transgenic Bt cotton field. Entomol. Know, 40: $160-163$.

Ludwig, J. A. and Reynolds, J. F. 1988. Statistical ecology: A primary methods and computing, New-York, 337pp.

Marguerite A. Rizk; Gihan M. E. Sallam; Nahla A.I. Abdel-Azim and Mona M.Ghallab. 2012. Spider occurrence in fields of some medicinal and ornamental plants in Fayoum, Egypt. Acarines (6): 41- 47.

Martin H. Schmidt; Indra Roschewitz; Carsten Thies and Teja Tscharntke. 2005. Differential effects of landscape and management on diversity and density of ground-dwelling farmland spiders $\mathrm{J}$. Appl. Ecol., 42(2): 281-287.
Nyffeler, M. 2000 a. Ecological impact of spider predation a critical assessment of Bristowe's and Turnbull's estimate. Bull. Br. arachnol. Soc., 11(9): 367-373.

Nyffeler, M. 2000 b. Killing power of the orbweaving spider argiope bruennichi (Scopoli, 1772) during a mass occurrence. Newsletter Br. arachnol. Soc., 89: $11-12$.

Nyffeler, M. and Benz, G. 1987. Spiders in natural pest-control. Journal of Applied Entomology, 103: 321-339.

Oberg, S. 2007. Diversity of spiders after spring sowing- influence of farming system and habitat type. J. Appl. Entomol., 131(8): 524-531.

Ostman, O.; Ekbom, B. and Bengtsson, J. 2003. Yield increase attributable to aphid predation by ground-living polyphagous natural enemies in spring barley in Sweden. Ecol. Econ., 45:149$/ 158$.

Schmidt, M. H.; Roschewitz, I.; Thies, C. And Tscharntke, T. 2005. Differential effects of landscape and management on diversity and density of ground-dwelling farmland spiders. J. Appl. Ecol., 42: 281-287.

Slingsby, D. and Cook, C. 1986. Prac Ecol. Macmillan, London: 213 pp.

Shuang-lin, J. and Bo-ping L. 2006. Composition and distribution of soil spider assemblages in three natural secondary forests in Ziwuling, Gansu. Zool. Res., 27 (6): 569- 574.

Southwood, T. R. E. 1978. Ecological methods with particular reference to the study of insect population. Chapman and Hall, London: 524 pp.

Symondson,W. O. C.; Sunderland, K. D. and Greenstone, M. H. 2002. Can generalist predators be effective biocontrol agents? Annu. Rev. Ent., 47: $561-594$.

Tahir, H. M. and Butt, A. 2009. Effects of different management practices and field margins on the abundance of ground spiders in rice ecosystems. Pakistan J. Zool., 41(2):85-93.

Weigmann, G. 1973. Zur Okologie der collemolen and Oribatiden in Gerenzhereich Land-Meer (Collembola, Insects, Oribatei, Acari). Z. iwss. Zool, Leipzig, 186 (3/4): 291-295.

Weis Fogh, T. 1948. Ecological investigation on mites and collembolan in the soil. Nat. Jutlant. , 1: $135-270$. 